\title{
Compensation is unnecessary for the perception of faces in slanted pictures
}

\author{
THOMAS A. BUSEY, NUALA P. BRADY, and JAMES E. CUTTING \\ Cornell University, Ithaca, New York
}

\begin{abstract}
In four experiments, we explored the perception of facial distortions seen in pictures viewed from the side or from above or below. In all four, however, we disguised the slant of the picture surface by using a double-projection technique that removed binocular and monocular cues: Faces were digitized, distorted to mimic a particular slant behind the image plane, cropped to a frame, and presented to viewers for their judgments. In the first experiment, we found that simulated rotations around a horizontal axis (pictures seen as if from above or below) created more noticeable distortions in faces than did simulated rotations around a vertical axis (pictures seen as if from the left or right). In the second experiment, pursuing a result from the first but with a between-subjects design, we found that pictured faces with a slant around a vertical axis of $22^{\circ}$ were seen as having no more distortion than unslanted faces. In the third experiment, we placed each image within a frame slanted either in the same way as or differently from the picture, and found no effect of frame. In the fourth experiment, we determined that viewers had little ability to match appropriately slanted frames with slanted pictures. Thus, we claim that part of the reason why one can look at moderately slanted pictures without perceptual interference is that the distortions in the image are subthreshold, or perhaps within the bounds of acceptability. These results contrast with the generally accepted theory that viewers mentally compensate for distortions in moderately slanted pictures.
\end{abstract}

Perhaps the most commonplace, but theoretically most surprising, aspect of the perception of realistic pictures, photographs, and cinema is their viewpoint-independent utility. That is, a viewer need not be directly in front of a picture or movie at the point from which it was composed (or shot) to enjoy it, to understand it, to admire it, or simply to look at it and make sense of what is seen.

Viewpoint-independent utility is theoretically surprising because the geometric layout of virtual space "behind" the picture surface is correct only for one viewpoint, variously called the station point (Bengston, Stergios, Ward, \& Jester, 1980; Cutting, 1986a; Gibson, 1979; Haber, 1978), the center of projection (Farber \& Rosinski, 1978; Kubovy, 1986), or the composition point (Cutting, 1988). (The line to this point from the center of the picture is called the principal ray, to which we will refer later.) Only by looking at a picture from the composition point is a cross section of the original, monocular optical array faithfully recreated. All other viewpoints induce optical deformations of greater or lesser extent. The issue at stake in this paper is how much slant-induced distortion produces a noticeable difference.

Although Leonardo (Richter, 1883/1970) and other 16th-century artists were concerned with particular deformations in pictures, it was La Gournerie (1859; see also

This research was supported by National Science Foundation Grant BNS-8818971. Correspondence should be sent to J. E. Cutting, Department of Psychology, Uris Hall, Cornell University, Ithaca, NY 148537601. We thank David Dunning for allowing us to gather the data of Experiment 2 in his class. T. A. Busey is now in the Department of Psychology at the University of Washington, Seattle, WA 98195.
Kubovy, 1986; Pirenne, 1970) who performed the first systematic global analysis of virtual space in pictures. He noted that when one's eye is not at the composition point, the virtual space behind the picture surface changes. When one is closer to a photograph, for example, than the focal length of the lens times the magnification, apparent depth should be compressed; when one is farther away, it should expand; and, most interestingly, when one is off to one side, it should undergo affine shear. Haber $(1978,1983)$, Hagen (1974), Hochberg (1986), and Sedgwick (1986) have discussed these issues; Bengston et al. (1980), Ellis, Smith, and McGreevy (1987), Goldstein (1979, 1987), Halloran (1989), Kraft and Green (1989), Rosinski and Farber (1980), and Rosinski, Mulholland, Degelman, and Farber (1980) have provided data showing effects of affine shears and compressions in pictorial space; and Cutting (1986a, 1987, 1989), Farber and Rosinski (1978), and, most notably, Kubovy (1986) and Pirenne (1970) have provided analyses of spatial distortions consistent with those of La Gournerie.

Yet there is a conundrum. In some circumstances, we are quite sensitive to distortions in pictures-particularly those involving comparisons of relations within a picture to the space of the viewers. In other circumstances, however, we are quite insensitive to distortions in pictures. We seem able to accept objects in slanted pictures for what they are supposed to be, and do not see them as distorted. How may we account for this conundrum?

\section{Three Attributes of Pictorial Space}

Goldstein (1987) discussed three attributes of virtual space in pictures. One, which we will call the perceived 
layout in a picture, is independent of observer position. That is, when Goldstein had observers recreate the spatial relations in the picture, they did so quite accurately regardless of their viewpoint within a $160^{\circ}$ arc in front of the picture. Another attribute of space, which we will call perceived orientation relative to the picture plane, is dependent on observer position, and conforms to the affine shears and compressions predicted by La Gournerie (1859; see Cutting, 1988). The third attribute, which we call perceived projective fidelity, is the focus of this paper and concerns distortions of objects by variation in the observer's viewpoint.

Consider a well-known example of these conjoined effects from World War I. In 1914, Alfred Leete constructed one of the most famous images of the 20th century-the British Secretary of State for War, Lord Kitchener, staring and pointing out from a recruiting poster with the caption "Your country needs you"' (see, e.g., Thompson \& Davenport, 1980). This image was quickly copied in the United States, so that in $1917 \mathrm{~J}$. M. Flagg produced the memorable image of Uncle Sam glaring and pointing out from an Army recruiting poster with the caption "I want YOU for the U.S. Army." What is important about these images is that Lord Kitchener and Uncle Sam appear relatively normal regardless of viewpoint (reflecting perceived layout and perhaps perceived fidelity in the projection of their faces), but their extended arms and forefingers follow the observer regardless of where he or she goes (reflecting different orientations relative to the picture plane).

\section{Compensation and Noncompensation Theories for the Perception of Slanted Pictures}

A major issue for perception of pictures at a slant, then, is how the perceiver accommodates the observer-relative deformations without loss of perceived object shape and perceived layout. Two types of theory have been proposed: those involving compensation and those not.

By far the most common explanation of picture perception at a slant is a two-stage theory relying on mental operations to rectify the Euclidean geometry of the original scene (Perkins, 1973; Pirenne, 1970; Rosinski \& Farber, 1980; Rosinski et al., 1980). We call this the standard compensation theory. The observer is thought to (1) register the image, and then (2) rectify it, compensating for distortions, according to the discrepancies between actual viewpoint and composition point. These discrepancies are usually thought to be computed on the basis of picture surface slant (Kubovy, 1986; Pirenne, 1970) derived from frame shape, graded binocular disparities, or other picture surface information. The compensation process can be thought of as akin to mental rotation (e.g., see Cooper \& Shepard, 1984).

A related theory of compensation invokes shape constancy to compensate for image distortions; we call it compensation-by-constancy theory. Wallach and Marshall (1986; Wallach \& Slaughter, 1988) promoted this idea because they were uncomfortable with the previous form of compensation, which makes picture perception inherently different from normal perception, doubling the perceptual process. Instead, they proposed that the observer's visual system (1) registers the image, and (2) then, by means of an automatic process involving shape constancy, renders objects normal in shape. Here again, however, the process uses screen slant information, and thus the theory seems empirically little different from the standard version of compensation theory.

We applaud Wallach and Marshall's (1986) discomfort with the idea of separating picture perception from the rest of perception, but we do not like two-stage theories. Our reasons are twofold. First, the perception of absolute slant of highly textured planes is not very good (Braunstein \& Payne, 1969; Epstein, 1981; Epstein, Bontrager, \& Park, 1962; Freeman, 1966; Perrone, 1980), and thus the basis on which slant corrections might be made seems shaky. Second, there is a more economical way-a simple one-stage notion, which we call subthreshold distortion theory.

According to Cutting (1987), the visual system might simply not discern distortions within certain limits of viewing angle. These limits generally accord with the measurements of Meister (1966); that is, within a cone of possible viewing space, measuring laterally from about $22^{\circ}$ to the left of the principal ray to $22^{\circ}$ to its right (as long as one is not too close to the image), no distortions will be noticed. Cutting (1987) provided support for this idea in experiments on the perception of cinema, in which he used a double projection technique. Adapted to our situation, it is shown schematically in Figure 1.

\section{A Double Projection Technique}

To understand this technique, imagine looking at a photograph at a particular slant. Imagine further an Albertian window (see Cutting, 1986a; Hagen, 1980) in front of the slanted picture, oriented so that it is orthogonal to the centric ray (the slanted ray passing from the eye to the center of the photograph). Next, through digital techniques, one can interchange these two projection surfaces, placing a simulated slanted surface behind the surface of the computer display. This technique has been outlined by Cutting (1986b), who has used it (Cutting, 1986a, 1987, 1989) for cinematic displays; here we will use it for pictures (see also Todd \& Reichel, in press, for a variant). Its major benefit is that it removes binocular and monocular cues to picture perception made on the basis of slanted surface information, but retains the image deformations due to slant.

Most important for the purposes of this paper is that Cutting (1987) found no difference in judgments of rigidity in rotating rectangular objects viewed at simulated projection slants of $0^{\circ}$ and $22.5^{\circ}$. Large differences appeared, however, with simulated slants of $45^{\circ}$. Thus, at least for cinema, no compensation is needed for one to perceive objects on slanted screens, as long as the slants are less than about $22^{\circ}$. Instead, such slants yield sub- 


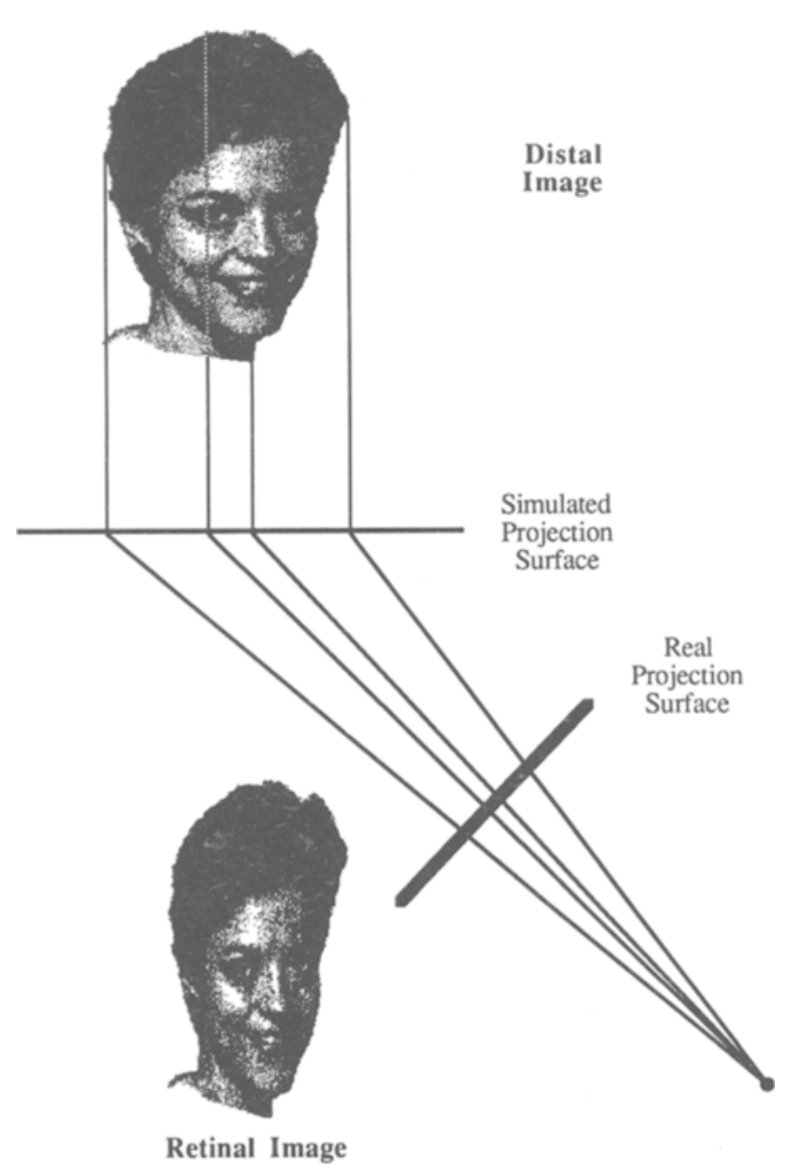

Figure 1. A schematic representation of the double-projection technique generating simulated picture slants without image slant information. The face at the top is seen without distortions, due to simulated slant of $0^{\circ}$; the face at the bottom shows the same face with distortions, due to a simulated slant of $+44^{\circ}$. The rays drawn depict a top view of the situation; the faces shown are, obviously, frontal views.

threshold distortions that simply are not noticed. Beyond $22^{\circ}$, however, various compensation processes may well occur.

Our purpose in this paper, then, is to extend the finding of evidence against compensation in slanted cinema to slanted pictures. That is, we believe that projective distortions in modestly slanted pictures, like those in cinema, are subthreshold, permitting normal picture perception processes to occur in roughly the same manner as normal perception occurs. For purposes of broadening the scope of this research program, we also altered our stimuli from being rectangular objects (see, e.g., Cutting, 1987; Perkins, 1972, 1973) to faces of people.

\section{EXPERIMENT 1 FACES IN PICTURES AS IF SEEN FROM LEFT, RIGHT, ABOVE, AND BELOW}

\section{Method}

Rather than take photographs of people, we combed newspapers and magazines for photographs of individuals suitable for digitiza- tion and storage as computer files on an Apple Macintosh microcomputer. We avoided culturally familiar faces; we also avoided those in advertisements because, in a study of the perception of distortions, we wanted to eliminate any materials that might already have been distorted without our knowledge. We selected photographs of four individuals from public interest articles, who seemed quite different and generally representative of people in the United States. For purposes of variability in the sample, two of the individuals were in full face and two in three-quarters profile. (We were unable to find suitable photographs of individuals in full profile.) None wore glasses; other characteristics are listed in Table 1.

The selected photographs were roughly $8 \times 12 \mathrm{~cm}$. They were digitized at a resolution of 28.4 pixels (picture elements) $/ \mathrm{cm}$, cropped to include only the face (removing all background), and scaled in size to fill the center of an Apple Macintosh monitor at a resolution of $215 \times 285$ pixels, or $7.4 \times 10.1 \mathrm{~cm}$.

The digitized images were manipulated to simulate pictures seen at a slant behind the computer screen, as is shown in Figure 1. The simulated viewing distance from the center of the slanted image was $.5 \mathrm{~m}$. Nine simulated picture slants were used: rotations of $0^{\circ}$ (where the center of the picture surface is orthogonal to the line of sight), and $\pm 22^{\circ}$ and $\pm 44^{\circ}$ around both horizontal and vertical axes passing through the center of the digital image. Sample stimuli for Individual A are shown in Figure 2. No images on the diagonal in Figure 2 (those with both horizontal and vertical rotations) were used, since they were much more easily seen as distorted. All rotations introduced aliasing artifacts into the bitmapped images; these were smoothed out by hand, using a mouse, and the corrected images were stored on disk file.

Each of the 36 images (4 individuals $\times 9$ rotations) was reduced for printing on an Apple LaserWriter II without bit smoothing (a process that interpolates bit patterns across adjacent pixels). Images with no rotation were reduced to $50 \%$ of their original size, those with $22^{\circ}$ rotations $52 \%$, and those with $45^{\circ}$ rotations $56 \%$. This differential reduction controlled roughly for changes in the projected area of faces introduced by differential rotation. The reduction process also doubled resolution ( 56.7 pixels $/ \mathrm{cm}$ ). The images were printed horizontally and vertically (in "landscape" and "portrait" formats), to control for possible artifacts introduced by the printing device. This yielded 72 prints that were then cropped to the same rectangular shape and size and placed in two notebooks

Table 1

Characteristics of the Individuals Whose Pictures Served as Stimuli, and Selected Results in the Four Experiments

\begin{tabular}{lllll}
\hline & \multicolumn{4}{c}{ Individuals } \\
\cline { 2 - 5 } & \multicolumn{1}{c}{ A } & \multicolumn{1}{c}{ B } & \multicolumn{1}{c}{ C } & \multicolumn{1}{c}{ D } \\
\hline Sex & Female & Female & Male & Male \\
Race & White & White & White & Black \\
Profile & 3/4 & Full face & 3/4 & Full face \\
Weight & Average & Heavy & Average & Thin \\
Approximate age & 30 & 30 & 60 & 20 \\
Relative hairlength & Short & Medium & Medium & Long \\
Headgear & None & None & Beret & None \\
Mean judgments for all rotations: & & \\
Experiment 1 & 3.6 & 4.0 & 3.4 & 4.1 \\
Experiment 2 & 3.4 & 4.0 & & 3.7 \\
Experiment 3 & 3.7 & 4.3 & 3.9 & 4.6 \\
Experiment 4 & 4.1 & 4.4 & & 3.6 \\
Mean judgments at & $0^{\circ}$ rotation: & & & \\
Experiment 1 & 1.9 & 2.5 & 1.7 & 1.8 \\
Experiment 2 & 2.1 & 3.3 & & 1.9 \\
Experiment 3 & 2.3 & 2.5 & 3.1 & 2.7 \\
Experiment 4 & 2.6 & 1.8 & & 1.4 \\
\hline
\end{tabular}

Note-Means for Experiments 1-3 are distortion judgments; those for Experiment 4 are matching judgments between face and frame slants. 


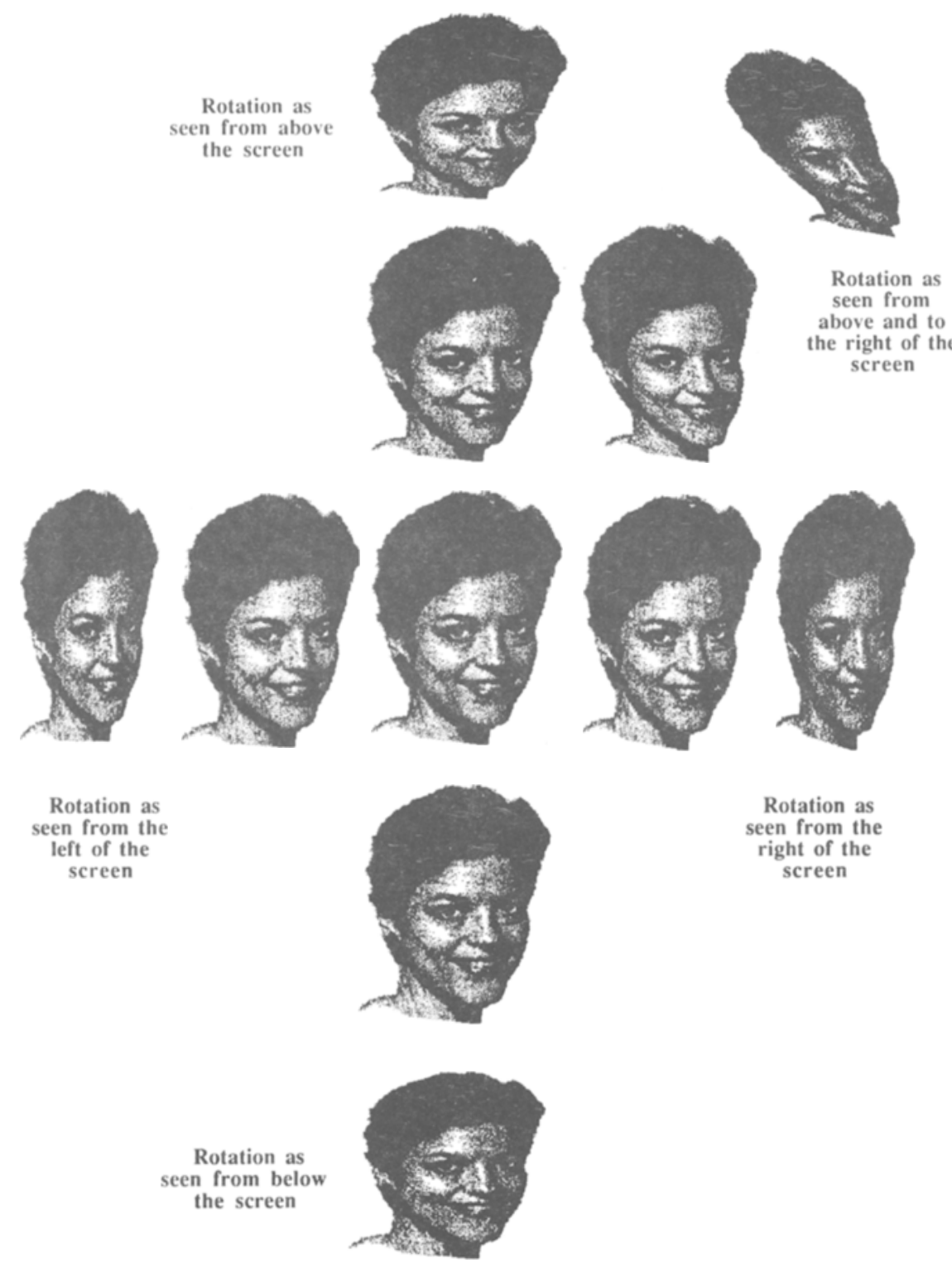

Figure 2. Images of Individual $A$, used in all four experiments. Those at the top underwent simulated rotation bringing the top of the head closer to the viewer; those at the bottom bring the neck closer; those at the left bring the left side of the image closer; and those at the right bring the right side closer. The middle image is the original stimulus with no simulated rotation; the four next to it (above, below, left, and right) have undergone $22^{\circ}$ simulated rotation; and the four farther out have undergone $44^{\circ}$ rotation. Two additional images not used in the experiments are shown, having undergone both horizontal and vertical rotations of $22^{\circ}$ and $44^{\circ}$.

of 36 images each. The images were suitably counterbalanced across notebooks. The order within a notebook was random under the constraints that no individual appear three times successively and no slant twice successively.

Sixteen members of the Cornell University community were paid $\$ 4.00$ each to peruse the two notebooks and make judgments about each image. Each participated individually; 8 looked at the notebooks in one order, 8 in the reverse order. The experimenter explained to them the nature of the distortions in pictures seen from above and below, and told them that the stimuli would mimic these. They were then told to make a judgment on a response sheet, indicating the general distortion seen in each image by using a scale from 1 to 7 , where 1 indicated no distortion and 7 the maximum distortion in the set. The task took about $30 \mathrm{~min}$.

\section{Results and Discussion}

We chose the four stimulus faces to represent the wide variety of people with whom our participants might be familiar. Before considering any analysis of the experimental variables, then, it is important to consider possible effects of our selection. Although there was a reliable main effect of stimulus face $[F(3,42)=20.03, p<$ 
$.0001]$ here, none occurred in the next two experiments. Selected data are shown in Table 1. Moreover, regression analysis showed that none of the variables listed at the top of Table 1 accounted for a significant amount of variance in the data (all $p s>.11$ ). Several interactions of stimulus face with experimental variables occurred, but explanation of these will be deferred until after presentation of the major results.

Of most interest is the relative distortion seen as a function of slant. There were no significant main effects for pictures seen from the right versus the left (with positive vs. negative rotations around the vertical axis), nor for those seen from above versus below (with positive vs. negative rotations around the horizontal axis). Thus, in this experiment, and in those that follow, we collapsed across stimuli with positive and negative rotations.

Major results are shown in the left-hand panel of Figure 3. Mean distortion judgments were lowest for pictures with no rotation (1.98), reliably lower than all others. Given the results of Cutting (1987), who found no difference in judgments of rigidity in cinematic displays seen with $0^{\circ}$ versus $22.5^{\circ}$ slants, we were a bit surprised by this result and pursued it in Experiment 2. However, as expected, images with $22^{\circ}$ rotations were seen as considerably less distorted than those with $44^{\circ}$ rotations $[2.70$ vs. $5.26 ; F(1,14)=130.3, p<.0001]$. Pictures with rotations around the vertical axis (left-right slants) were seen as less distorted (3.56) than those around the horizontal axis $[4.43 ; F(1,14)=49.8, p<.0001]$. Vertical rotations produce incrementally slimmer faces and horizontal rotations produce rounder faces; ratios of facial shape will be the focus of the next section.

\section{Measurements Associated with \\ Perceived Distortions in Faces}

When looking at the range of stimuli, it became apparent to us some of the differences between vertical and horizontal rotations, and some of the interactions involving different individual faces, could be explained by facial proportions in slanted and unslanted faces. To analyze this possibility we selected Individuals B and D (see
Table 1) to examine further. Of our four individuals, these two had the widest (B) and thinnest (D) faces of the set, and they were the only ones appearing full face and thus facilitating measurements. On all nine images of each individual, we measured the width of the face across the cheekbones and the height of the face from the chin to the top of the eyebrows. We then made a ratio of the two $(\mathrm{H} / \mathrm{W})$, and $\log$ transformed that value. Resultant values greater than zero indicated elongated faces (here as if seen from the side) and those less than zero indicated squashed faces (here as if seen from above or below). We then plotted distortions seen as a function of $\log (\mathrm{H} / \mathrm{W})$, which are shown in Figure 4.

Four trends can be seen. First, the data can be fit roughly by the inverse of a normal distribution. Second, the minimum of that function is at a point slightly greater than 0.0 , indicating that a best (least distorted) face is relatively thin compared to its height. Third, the left-hand limb of the function is higher than the right-hand limb, indicating, as before, that horizontal rotations create more noticeable distortions than vertical ones do. And fourth, although the two individuals selected had different facial ratios at each slant, their data both generally fit on the function, with Individual D's data slid rightward along the function as compared with Individual B's at all rotations.

Our results support the idea that people have a rough template of a generalized human face stored in memory, against which comparisons with the image can be made (Brennan, 1985; Rhodes, Brennan, \& Carey, 1987). For comparison with our data, we calculated the $\log (\mathrm{H} / \mathrm{W})$ values for other faces and samples of faces, also shown in Figure 4 along the abscissa. Values are given for a prototypic face from Dürer (1528/1972, p. 222) marked D, for the sample face from Rhodes et al. (1987, Figure 5) marked R, and for schematic faces used by Brunswik (1956) whose range is delimited by the two Bs. As one can see, these correspond reasonably well to the best faces in our set.

Given the rough template of a face, then, a constancy process might seem to account for one aspect of our results: "We know what shape any object really has be-
Experiment 1

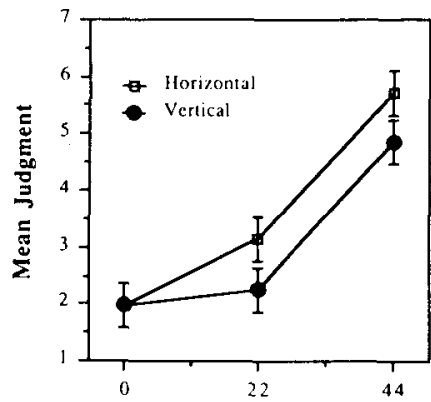

Experiment 2

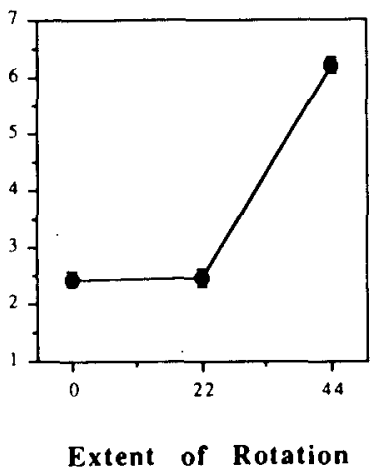

Experiment 3

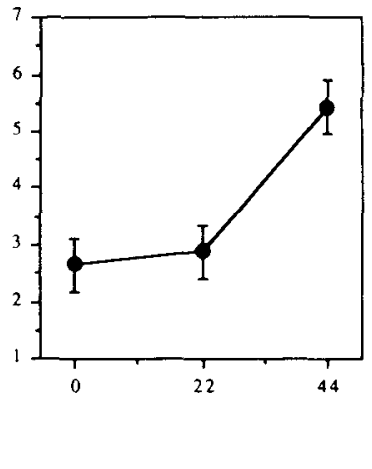

Figure 3. Judgments of facial distortion in the first three experiments, as a function of slant, in degrees of rotation. 


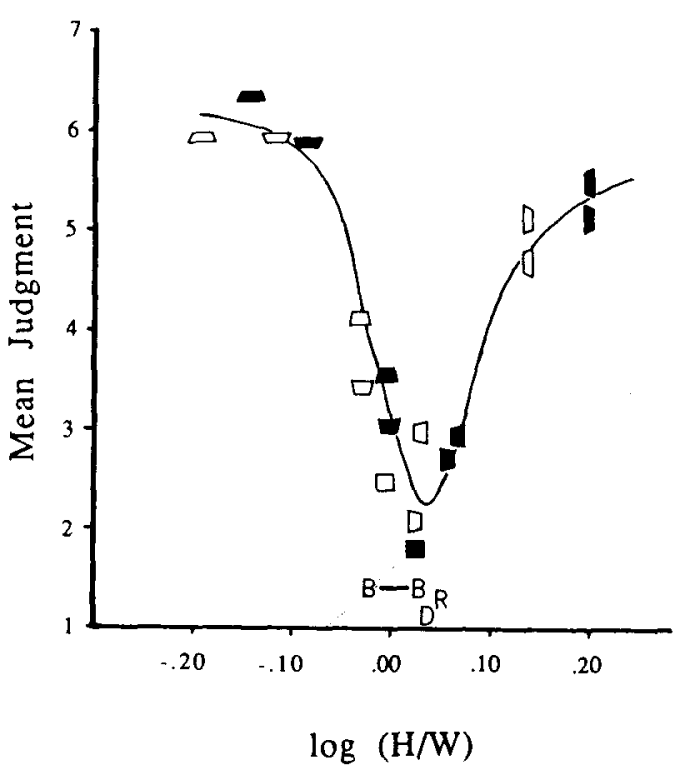

Figure 4. Judgments of facial distortion in Experiment 1 as a function of the $\log$ (height/width) of the face for Individuals $B$ and $D$. The location of datapoints is indicated by icons representing the slant of a frame, had the frame appeared with the face (which it did not in this experiment). Square frames indicate no slant, thin frames $44^{\circ}$ slant, and intermediate frames $22^{\circ}$ slant. Open icons represent data for Individual B, closed icons for Individual D. Mean standard error of the mean for all datapoints is 0.22 . The continuous function was fit by hand. Additional values along the abscissa are shown taken from measurements of faces appearing in Brunswik (1956) (B), Dürer (1528/1972) (D), and Rhodes et al. (1987) (R).

cause of our previous experience with it" (Hochberg, 1972, p. 514). However, this is not the constancy process proposed by Wallach and Marshall (1986), which is informed by cues of slant.

\section{Overview}

Three results stand out. First, rotations around the vertical axis are less noticeable than those around the horizontal axis. Second, rotations of $22^{\circ}$ seem to introduce modest but discernible distortions beyond those seen in unrotated pictures, whereas rotations of $44^{\circ}$ introduce clearly noticeable distortions. Third, there may be an optimal face shape (perhaps part of a facial schema) against which viewers may make comparisons. In the next experiment, we pursued the second result in a between-subjects design.

\section{EXPERIMENT 2 \\ FACES IN ISOLATED PICTURES WITH SIMULATED SLANT}

We were worried about the slight but statistically reliable difference in judgments of pictures slanted $0^{\circ}$ versus $22^{\circ}$ in Experiment 1. Cutting (1987) found no differences in the two slants. To be sure, the two experimental situations are quite different: Experiment 1 concerned the perception of faces on slanted pictures, and the experiments of Cutting (1987) concerned the perception of rotating rectangular solids on slanted screens. Thus, the former result concerns frozen images, and the latter result concerns cinematic ones. Nonetheless, since Cutting (1987) argued that his effect was likely due to the inability of the human visual system to discern distortions induced by moderately slanted screens, we expected that the result should generalize to frozen images.

One important methodological difference in the two experimental situations, however, is that here the faces are distinctive enough so that participants could remember the set of images of each face over the course of the experiment. Cutting's (1987) rotating rectangular solids were all unremittingly alike, and no single frame was ever available to be remembered. Thus, participants in Experiment 1 could make comparisons within the set of images of each face, and then make judgments based at least partly on that information. For our purposes, memory for a given face shape is irrelevant to the task under study. To overcome this possible complication, we conducted a partial replication of Experiment 1 using a between-subjects design, such that no participant saw more than one rendition of any stimulus face.

\section{Method}

Six different types of four-page booklets were prepared. Three of the four faces from Experiment 1 were used as test stimuli. In that experiment, Individual $C$ had shown the least difference in distortion judgments between $0^{\circ}$ and $22^{\circ}$ pictures, so we removed him from the test set to serve as an example stimulus. This procedure effectively made the test of our hypothesis (that there should be no difference between rotations of $0^{\circ}$ and $22^{\circ}$ ) more difficult. Two images of Individual $\mathrm{C}$, with $0^{\circ}$ and $+44^{\circ}$ slants (again, positive slant means that the right edge is closer to the viewer), were placed on the cover page of each booklet, with an indication that the $0^{\circ}$ stimulus should receive a distortion rating of 1 and the $+44^{\circ}$ stimulus a rating of 7 .

The next three pages differed across the six booklet types. In each booklet type, there was one $0^{\circ}$, one $22^{\circ}$, and one $44^{\circ}$ stimulus rotated around the vertical axis, each on a separate page. Half the booklet types used one $+22^{\circ}$ slant and one $-44^{\circ}$ slant, the other half $-22^{\circ}$ and $+44^{\circ}$. In one third of the booklet types, Individual $\mathrm{A}$ was assigned the $0^{\circ}$ slant, Individual B the $22^{\circ}$ slant, and Individual $\mathrm{C}$ the $44^{\circ}$ slant; in another third, the assignment was $\mathrm{B}$ : $0^{\circ}, \mathrm{C}: 22^{\circ}$, and $\mathrm{A}: 44^{\circ}$; and in the final third, it was $\mathrm{C}: 0^{\circ}, \mathrm{A}$ : $22^{\circ}$, and B: $44^{\circ}$. Finally, within each type of booklet, the order of the last three pages was haphazard. All images were laser printed in "portrait" format.

The booklets were ordered, repeating in sequence through the six types, and handed out in serial fashion as part of an exercise in a Psychology and Law class at Cornell University. Five minutes at the end of the class was devoted to test instructions, judgments, and discussion. Again, the participants were told that distortions mimicked those in pictures seen from the side. The participants wrote responses on the booklets, using the 1 to 7 scale. A total of 328 booklets were returned, but with unequal numbers per booklet type. To equalize them, 11 were randomly selected from the largest groups and dropped, and an additional 13 students from the same population, but not members of the same class, were recruited to fill in the smallest groups. After this adjustment procedure, each of the six booklet types had 55 sets of judgments.

\section{Results and Discussion}

There were no differences across the three stimulus faces, nor again was there any effect of the variables shown in Table 1. Most important for the hypothesis un- 
der test, as shown in the central panel of Figure 3, is that there was no difference between $0^{\circ}$ and $22^{\circ}$ rotations $[2.44$ vs. $2.46 ; F(1,329)=0.04, p>.85]$. As expected, and as before, there was a large difference between $22^{\circ}$ and $44^{\circ}$ rotations $[2.44$ vs. $6.20 ; F(1,329)=1550.9$, $p<.0001]$. Thus, distortions introduced in pictures slanted around the vertical axis up to at least $22^{\circ}$ are insufficient to be noticed, corroborating the results of Cutting (1987).

In the first two experiments, we explored the notion that images in slanted pictures are somehow rectified by taking into account the degree of picture surface slant. We sought disconfirming evidence, testing the amount of distortion seen in slanted images without surface slant information. Up to $22^{\circ}$ around the vertical axis, we found distortions to be unnoticed. Thus, no compensation process is necessary for images in pictures slanted to this extent or less. In our next two experiments, we explored the sufficiency of slant information by providing frames around each image that either matched or mismatched the slant of the image itself.

\section{EXPERIMENT 3 JUDGMENTS OF FACIAL DISTORTION IN SLANTED PICTURES WITH MATCHING AND MISMATCHED SLANTING FRAMES}

Since previous theories of slanted picture perception (Kubovy, 1986; Pirenne, 1970; Rosinski et al., 1980) had explicitly incorporated frame shapes to rectify slanted images, we presented to viewers images with appropriate and inappropriate frames around them.

\section{Method}

The four faces of Experiment 1 were used here. As in Experiment 2 , instead of using both vertical and horizontal rotations, we concentrated on vertical rotations only, using $0^{\circ}, \pm 22^{\circ}$, and $\pm 44^{\circ}$ rotations. Crossed with these were five frame slants: $0^{\circ}, \pm 22^{\circ}$, and $\pm 44^{\circ}$. These selections and manipulations created 100 stimuli: 4 faces $\times 5$ image slants $\times 5$ frame slants. Examples of Individual $A$ are shown in Figure 5.

Rather than laser print these images, we presented them in random sequence on an Apple Macintosh monitor. The cropping of an image within a particular frame outline occurred automatically prior to the image's appearing on the screen. The frames subtended about $4^{\circ}$ visual angle, and the monitor about $15^{\circ}$, almost four times as large in linear extent. Ten different students from the Cornell community participated. Each was told about the nature of the distortions in the stimuli, and was given 25 practice trials to get used to the scale ( 1 to 7 as before, but this time entered on the console) and the range of stimuli. Participation time was about $15 \mathrm{~min}$, and each individual was paid $\$ 2.00$ for this effort.

\section{Results and Discussion}

Regression analyses again showed that none of the factors listed in Table 1 accounted for any systematic variance in the results, and as in Experiment 2, there were no reliable differences across the four stimulus faces.

Most surprising, however, was that there was no effect of the slant of the frame $[F(4,36)=1.02, p>.41]$. More particularly, frames with slants that matched pic-
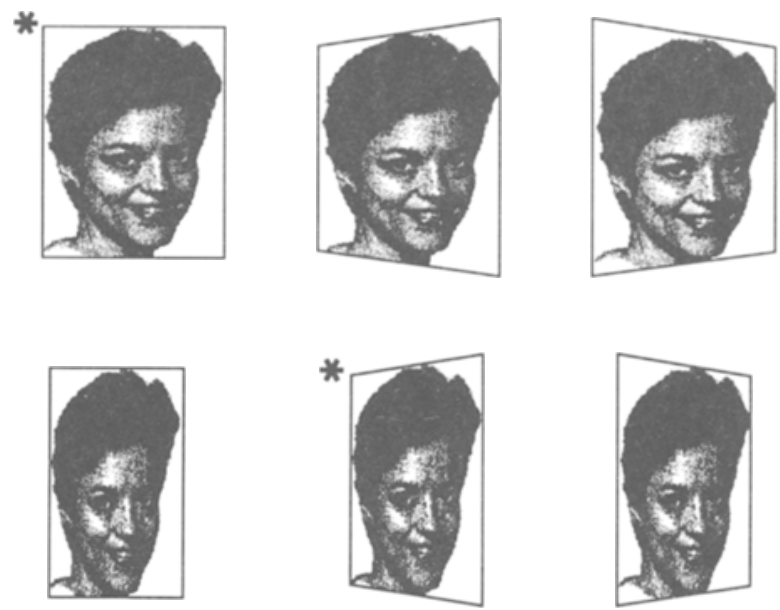

Figure 5. Selected matched and mismatched frames with images of Individual A, as used in Experiment 3. The top three have $0^{\circ}$ rotation of the face and $0^{\circ},+22^{\circ}$, and $-22^{\circ}$ rotations of the frame. The bottom three have $+44^{\circ}$ rotation of the face and $0^{\circ},+44^{\circ}$, and $-44^{\circ}$ rotations of the frame. Thus, the two images marked with an asterisk have matching face and frame slants.

ture slants did not decrease the apparent distortion seen in the face over those that mismatched $[4.05$ vs. 4.15 , respectively; $F(1,9)=0.55, p>.47$ ]. The top panel of Figure 6 shows the results for all matched and mismatched faces and frames, collapsing across positive and negative slants. As is apparent, our participants could successfully filter out frame information around a face. This result, of course, cannot be interpreted to indicate that frame shape is not used in normal picture perception, but the fact that frame shape is so handily ignored in this experiment impugns any compensation theory's assumption of a tight bond between frame shape and image distortion.

For comparison with previous findings, the further condensed results are shown in the right-hand panel of Figure 3. That is, images with $0^{\circ}$ slant (irrespective of frames) were seen as least distorted (2.65); those with $22^{\circ}$ slant were seen as somewhat more distorted (2.88); and those with $44^{\circ}$ slant were seen as considerably more distorted (5.43). As in Experiment 1, all pairwise differences are reliable ( $p \mathrm{~s}<.001$ ), but given the results of Experiment 2 , the difference between $0^{\circ}$ and $22^{\circ}$ is likely due to the participants' familiarity with the entire data set.

\section{EXPERIMENT 4 \\ JUDGMENTS MATCHING SLANTED FRAMES WITH SLANTED FACES}

Experiment 3 showed no effect of frames on the perception of faces seen at a slant. Unfortunately, there are at least two relatively uninteresting reasons for this noneffect. First, the presentation of the wide selection of matching and mismatching frames could easily have caused our participants to ignore frames; frames were literally not relevant to the task. With less experimental experience, frame effects might be obtained. Second, the moni- 


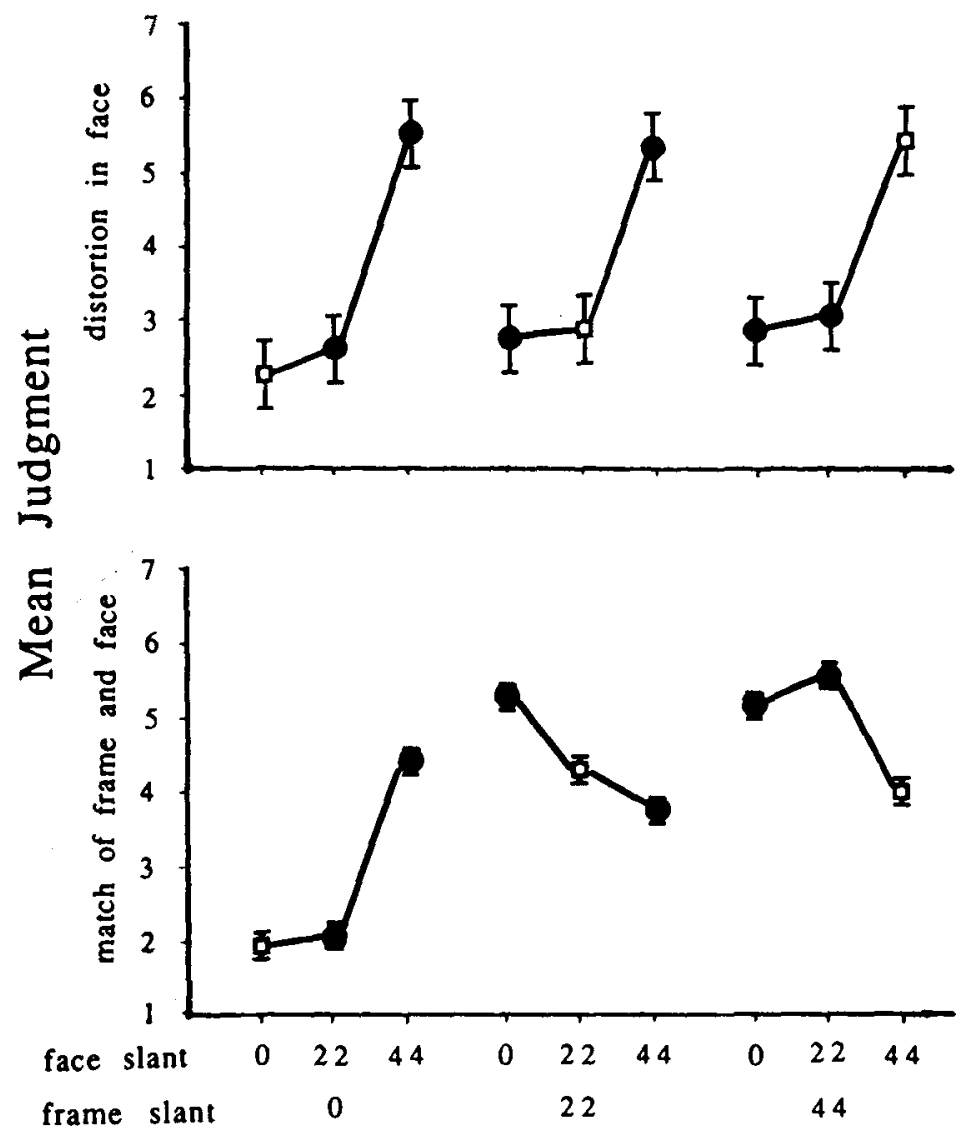

Figure 6. Judgments for matched and mismatched frames and faces in Experiments 3 and 4. Those in Experiment 3 were for facial distortions only; those in Experiment 4 indicate whether observers thought frame and face matched or mismatched. Open squares correspond to matching face and frame slants, closed circles to mismatching face and frame slants.

tor of the Apple Macintosh microcomputer (about $15^{\circ}$ ) may have served as an additional frame around the slanted frames $\left(4^{\circ}\right)$ of the faces. It is possible, although we think it unlikely, that the unslanted nature of the considerably larger frame dominated the slanted frames drawn on the screen. This experiment was conducted, in part, to counter these possibilities.

In addition, the results of Experiment 3 are silent on the issue of whether observers can use information about slant from frame shape; the previous results simply show that they did not use it. Moreover, evidence of Goldstein (1987) and Halloran (1989) suggest some ability to use frame shape information, although that ability may not be highly refined (see, e.g., Freeman, 1966). Thus, this time we had observers try to distinguish between frames and faces that matched in slant from those that mismatched. If they were reasonably accurate at this task, there would be some basis for believing that a compensation process can take place; on the other hand, if they were reasonably inaccurate, this result should be taken as fur- ther evidence against any two-stage theory of slanted picture perception.

\section{Method}

To counter the possibility that our previous participants simply learned to ignore frames, we used a between-subjects design with few stimuli presented to each participant, as in Experiment 2. To counter the possibility that a superordinate, unslanted frame dominated slanted frames, the images were laser printed (as in Experiments 1 and 2), and placed three to a sheet in a haphazard fashion.

Nine different response sheets were prepared, each with the three faces from Experiment 2. Twenty-seven pairs of faces and frames were matched, and we will designate them with pairs of numbers separated by a slash; thus, $0 / 22$ indicates an unslanted face with a frame slanted by a vertical rotation of $22^{\circ}$. Only positive vertical rotations were used (those with the right edge simulated to be closer to the observer). The groups balanced the three face slants $\left(0^{\circ}, 22^{\circ}\right.$, and $\left.44^{\circ}\right)$, the three frame slants $\left(0^{\circ}, 22^{\circ}\right.$, and $\left.44^{\circ}\right)$, and the three faces. Three groups rated the $0 / 0,22 / 44$, and $44 / 22$ stimuli; three groups the $0 / 22,22 / 0$, and $44 / 44$ stimuli; and three groups the $0 / 44$, $44 / 0$, and $22 / 22$ stimuli. Within each triad of groups, the three faces were rotated across the three pairings of face/frame slants. 
Members of a perception class at Cornell participated as part of a course project: 216 returned completed forms, 12 of which were dropped, and 5 more forms were added (filled out by students from the same population) to balance the number of participants at 23 per group. Participants were shown examples of blank picture frames with $0^{\circ}, 22^{\circ}$, and $44^{\circ}$ slants. They were then shown, and told about, the nature of pictures seen from the side. They were asked to judge the extent to which the three faces and frames in their booklets matched in degree of slant, using a 1 to 7 scale, with 1 indicating a perfect match $\left(0^{\circ}\right)$ and 7 the largest possible mismatch $\left(44^{\circ}\right)$.

\section{Results and Discussion}

Since all viewers were given booklets with frames of $0^{\circ}, 22^{\circ}$, and $44^{\circ}$, they had no difficulty in determining which slant was which. If viewers are able to use frame slant information to rectify distortions in images seen from the side, they ought to be able to compensate for the facial distortions by using the differential slant information. Relative success at rectifying the distortions should be reflected in low ratings; nonsuccess in higher ratings.

The results are shown in the bottom panel of Figure 6, again parsed into three groups according to the slant of the frame. There was a large effect of frame slant $[F(2,594)=74.9, p<.0001]$, seen in the relatively low values given for unslanted frames (2.78) and the higher values given for frames with $22^{\circ}$ and $44^{\circ}$ slant $(4.43$ and 4.87). This result indicates either that any attempt at compensation only makes the faces look worse, or that the slants of all faces generally seem to be less than the slant of the frame. In opposition to the frame effect, for facial slant there was no effect $[F(2,594)=0.21, p>.80]$. That is, overall, the slant of the face did not contribute to the judgment. Together, these two main effects suggest, despite instructions, that judgments were not made about the matching of face slant and frame slant, but were based largely on the slant of the frame.

As should be obvious, however, the face slant $\times$ frame slant interaction was highly reliable $[F(4,594)=33.3$, $p<.0001]$, and this interaction is worth further consideration. The left-hand group of data, that for $0^{\circ}$ frame slants, matches the data in the three panels of Figure 3 and that in the panels of Figure 6 above it. That is, judgments matching facial slants to frame slants within an unrotated frame mimic the judgments for distortions seen in faces. In particular, there was no reliable difference between $0 / 0$ and $0 / 22$ stimuli $[t(136)=0.56, n . s$.$] , but there was a$ large difference between both of them and $0 / 44$ stimuli $[t \mathrm{~s}(136)>7.9, p \mathrm{~s}<.001]$. That these results mimic the earlier findings makes sense: The extent of mismatch between face and frame can only be determined through a judgment of facial distortion.

The middle and right groups of data reveal more complexity, and they are clearly different from those plotted above in Figure 6. Despite the complexity, however, two general points can be made. First, mean results for all frame slants of $22^{\circ}$ and $44^{\circ}$ differ little [but reliably; $t(413)=6.3, p<.001]$ from the midrange of the scale
( 4 vs. 4.6 ), and the mean of the results for $0 / 0$ and $0 / 22$ are quite different (1.99) from all others (4.59). The latter simple contrast accounts for $90 \%$ of the variance in the mean data, and when partialed out, it renders nonsignificant the main effect of frame slant. Although there are some reliable differences among these seven scores, they indicate systematic regression toward the middle of the scale and little confidence on the part of the participants in performing the task.

Second, the reliable differences in these data cannot be predicted well by any theory that is based on the ability to match face and frame slants. For example, eliminating those three groups who rated $0 / 0$ stimuli (which had a large difference between same-slant [1.93] and differentslant [4.70] judgments), there were no differences among the other six groups. That is, same-slant stimuli $(22 / 22$ and $44 / 44)$ were rated about the same $(4.04)$ as were different-slant stimuli $[4.19 ; F(1,132)=0.47, p>.48]$.

Finally, there were large differences among the ratings for the nine groups $[F(8,198)=7.6, p<.001]$, differences among the three faces $[F(2,594)=29.4, p<.001]$, as is shown in Table 1 , and an interaction of the three faces with frame slant $[F(4,594)=33.3, p<.001]$, as has been discussed earlier with respect to Experiment 1 .

A traditional qualm and a response. We claim that these results demonstrate little ability on the part of subjects to match the perceived slants of picture frames and their contents, and hence little ability to use frame slant information in slanted picture perception. In particular, when the frame is unslanted (see the bottom-left panel of Figure 6), the pattem of results is not different than it is when no frame is presented; and when the frame is slanted (the other lower panels), the results show no systematic pattern.

One can counter this claim on theoretical grounds, using a distinction often found in illusion and constancy research (see, e.g., Hershenson, 1989). That is, there may be a difference between the registration of frame slant and the perception or even knowledge of frame slant. Registered slant can be thought of as information inaccessible to conscious decision making that is used automatically. Moreover, registered slant might be used for compensation with slanted pictures, and perceived or known slants might have been used in the present task.

The distinction between registered and perceived information is nonparsimonious and subject to difficulties. Nonetheless there is some evidence to support it (Hochberg, 1972). In our experimental context, however, there are at least three problems with this distinction and its application. First, if the registration and use of frame slant information is unconscious and automatic, the results of Experiment 3 ought to have been quite different, showing effects of frame slant. There were none. Second, the results of Experiment 3 should have been different from those of Experiments 1 and 2, where no frames were used. Yet the results are the same. Third, the results of Experi- 
ment 4 indicate that viewers cannot explicitly use frame slants; if they cannot explicitly use this information, there would seem to be little hope that viewers could implicitly use it. Thus, we think that the distinction provides little insight into our results.

\section{GENERAL DISCUSSION}

The results of these four experiments bear on three critical issues for general theories of picture perception: the tolerance for distortion due to slant, the stages involved in the process of perceiving slanted pictures, and the perceptual coordination of various attributes of pictorial space.

\section{Distortions of Projection when}

\section{a Picture Is Viewed from the Side}

How should one think about tolerance for distortions in slanted pictures? Following Meister (1966), we think that the best measure is that which concerns the shrinkage in the dimension orthogonal to the axis of picture rotation. When a picture is seen from the right or the left, the effective horizontal compression along the middle of the image is the cosine of the degree of slant. Thus, from a sufficient viewing distance, a picture slanted at $22^{\circ}$ has its width reduced to about $93 \%$, for a $7 \%$ reduction. Our data with simulated slants show that this is subthreshold, or at least within the bounds of normality. When a picture is slanted $44^{\circ}$, its width is reduced to about $72 \%$, for a $28 \%$ reduction. To be sure, all slants around a vertical axis will cause some $y$-axis expansion in the image to the side of the picture brought closer to the observer, and shrinkage in the image on the other side; but given general asymmetries in typical human faces, these other perspective effects may not be noticeable.

\section{Against Two-Stage Theories of Picture Perception}

Theories of picture perception must be viewpointgeneral; that is, they must (1) admit the general unacceptability of extreme viewpoints that create large slants (e.g., an angle of greater than about $45^{\circ}$ between the principle and centric rays) and (2) embrace the acceptability of less extreme viewpoints that create modest slants (less than about $22^{\circ}$; Meister, 1966). The latter phenomenon is usually thought to be accomplished by a separate perceptual stage in which the image is rectified of its slant-based distortion from information available about screen slant. Compensation theories, however, typically do not address the former phenomenon-in this case, why rectification does not seem to work on pictures with more extreme slants.

Beyond this logical problem, two-stage theories involving processes compensating for slant are unnecessary and insufficient to explain our data. That is, taken together, the results of Experiments 1 and 2 showed that facial distortions in pictures with simulated slants of up to $22^{\circ}$ (rotated around the vertical axis) are unnoticed, and that these faces are functionally indistinguishable from unslanted ones. Thus, they are simply not in need of compensation. The results of Experiment 3 demonstrated, at minimum, that slanted and unslanted frames around faces are easily ignored and do not provide compelling context for slant. Moreover, the results of Experiment 4 demonstrated that frame slant information is largely unusable in relation to projective distortions.

But could a theory of compensation still operate? Yes, when one views a picture or cinema from an angle beyond $22^{\circ}$ to the side or even from above or below within $22^{\circ}$, compensation processes might be necessary. However, we suggest that they would seldom be used. Our rationale is twofold. First, as has been shown by Meister (1966), few theaters allow many seats in areas beyond $22^{\circ}$ from the principal ray, and even when they do, few people (other than the present authors, perhaps) sit there by choice. Second, when viewing photographs, people will almost always physically rotate the picture or bring themselves within a cone of sight less than $22^{\circ}$ from the principal ray. Thus, compensatory processes are either inadequate or arduous to invoke. People, given a choice, will choose not to try to use them.

\section{On Coordinating Attributes of Pictorial Space}

In our introduction, we noted Goldstein's (1987) tripartite analysis of pictorial space: (1) The perceived layout of the space within a picture is not affected by extreme viewer position within $70^{\circ}$ of the principal ray, but (2) the perceived projective distortions of its objects and (3) the perceived orientation of objects within the picture relative to the picture plane are quite position-sensitive. Cutting (1988) tried to argue that the latter two attributes are difficult to disentangle and may be handled by the same principles, but the data presented here and in Cutting (1987) suggest that sensitivity to viewer position does not affect the perceived projective fidelity within $22^{\circ}$ of the principal ray as much as does orientation relative to the picture plane, which is sensitive to within a few degrees.

Orientation relative to the picture plane is an interesting phenomenon well worth a good explanation. See Cutting (1988) for one attempt; see Ellis et al. (1987) and Halloran (1989) for others. Nonetheless, it is a phenomenon of the relation between observers and pictures; it is not a phenomenon of picture perception. To the contrary, Goldstein's other attributes-perceived layout and perceived projective distortions-are very much phenomena of picture perception, and it is these that must be coordinated in the perception of the contents of pictures.

\section{A CONCLUSION}

We have presented a theory of the perception of slanted pictures, but not a general theory of picture perception. Nonetheless, such a theory must embrace the idea that at different times and different places, two attributes of pictorial space are important-perceived layout and perceived projective distortion. If a perceiver wants to know 
generally what the picture is of, simply by perceiving the layout of objects within the picture, then almost any viewpoint will do. Perceived layout does not depend on viewer position. If, on the other hand, one wants to discern the contents of a picture, one must bring the slant of the picture to $22^{\circ}$ or less (either by moving oneself or by moving the picture), making projective distortions subthreshold, or at least acceptable. (There is, of course, nothing particularly special about $22^{\circ}$, and the effect may persist out to $30^{\circ}$ or more.) Thus, a theory of picture perception must also be a theory of the viewer's behavior in front of pictures.

\section{REFERENCES}

Bengston, J. K., Stergios, J. C., Ward, J. L., Jester, R. E. (1980) Optic array determinants of apparent distance and size in pictures. Journal of Experimental Psychology: Human Perception \& Performance, 6, 751-759.

Braunstein, M. L., Payne, J. W. (1969). Perspective and form ratio as determinants of relative slant judgments. Joumal of Experimental Psychology, 81, 584-589.

Brennan, S. E. (1985). The caricature generator. Leonardo, 18. 170-178.

Brunswik, E. (1956). Perception and the representative design of psychological experiments. Berkeley: University of California Press.

COOPER, L. A., \& SHEPARD, R. N. (1984). Turning something over in one's mind. Scientific American, 251(6), 106-114.

Cutring, J. E. (1986a). Perception with an eye for motion. Cambridge, MA: Bradford Books/MIT Press,

Cutring, J. E. (1986b). The shape and psychophysics of cinematic space. Behavior Research Methods, Instruments, \& Computers, 18, 551-558.

Cutring, J. E. (1987). Rigidity in cinema seen from the front row, side aisle. Journal of Experimental Psychology: Human Perception \& Performance, 13, 323-334.

Curnivg, J. E. (1988). Affine distorions of pictorial space: Some predictions for Goldstein (1987) that La Gournerie (1859) might have made. Joumal of Experimental Psychology: Human Perception \& Peformance, 14, 305-311.

Cutring, J. E. (1989). On the efficacy of cinema, or what the visual system did not evolve to do. In S. Ellis, M. Kaiser, \& A. Grunwald (Eds.), Spatial displays and spatial instruments (NASA Conference Publication 10032, pp. 17:1-8). Moffett Field, CA: NASA.

Dürer, A. (1972). The human figure: The Dresden sketchbooks (W. L Strauss, Ed. \& Trans.). New York: Dover. (Original work published 1528)

Ellis, S. R., Smith, S., McGreevy, M. W. (1987). Distortion of perceived visual directions out of pictures. Perception \& Psychophysics, 42, 535-544.

Epstein, W. (1981). The relationship between texture gradient and perceived slant-in-depth: Direct or mediated? Perception, 10, 695-702.

Epstein, W., Bontrager, H., \& Park, J. (1962). The induction of nonveridical slant and the perception of shape. Joumal of Experimental Psychology, 63, 472-479.

FARBer, J., \& Rosinski, R. R. (1978). Geometric transformations of pictured space. Perception, 7, 269-282.

Freeman, R. B. (1966). Absolute threshold for visual slant: The effect of stimulus size and retinal perspective. Journal of Experimental Psychology, 71, 170-176.

Gibson, J. J. (1979). The ecological approach to visual perception. Boston: Houghton Mifflin.

GolDsteIN, E. B. (1979). Rotation of objects in pictures viewed at an angle: Evidence for different properties of two types of pictorial space. Joumal of Experimental Psychology: Human Perception \& Performance, $5,78-87$

Goldstein, E. B. (1987). Spatial layout, orientation relative to the observer, and perceived projection in pictures viewed at an angle. Journal of Experimental Psychology: Human Perception \& Performance, 13, 256-266.
Haber, R. N. (1978). Visual perception. Annual Review of Psychology, 29. 31-59

HABER, R. N. (1983). Stimulus information and processing mechanisms in visual space perception. In J. Beck. B. Hope. \& A. Rosenfeld (Eds.), Human and machine vision (pp. 157-235). New York: Academic Press.

Hagen, M. A. (1974). Picture perception: Toward a theoretical model. Psychological Bulletin, 81, 471-497.

HAGEN, M. A. (Ed.) (1980). The perception of pictures $(2$ vols.). New York: Academic Press.

Halloran, T. O. (1989). Picture perception is array-specific: Viewing angle versus apparent orientation. Perception \& Psychophysics. 45, 467-482

HeRshenson, M. (Ed.) (1989). The moon illusion: An anomaly of tisual space perception. Hillsdale, NJ: Erlbaum.

HoснвеRG, J. (1972). Perception II: Space and movement. In J. W Kling \& L. A. Riggs (Eds.), Woodworth \& Schlosberg's Experimental psychology (3rd ed.; Vol. 1.,pp. 475-550). New York: Holt. Rinehart \& Winston.

HOCHBERG, J. (1986). Representation of motion and space in video and cinematic displays. In K. R. Boff, L. Kaufman, \& J. P. Thomas (Eds.), Handbook of perception and human performance (Vol. 1, pp. 22:1-64). New York: Wiley.

Kraft, R. N., \& Green, J. S. (1989). Distance perception as a function of photographic area of view. Perception \& Psychophysics, 45. 459-466.

KuBovy, M. (1986). The psychology of perspective and Renaissance ant. London: Cambridge University Press.

LA Gournerie, J. De. (1859). Traité de perspective linéare contenant les tracés pour les tableaux, plans et courbes, les bas-reliefs et les décoration théatrales, avec une théorie des effets de perspective [Treatise on linear perspective containing drawings for paintings, architectural plans and graphs, bas-reliefs, and theatrical set design, with a theory of the effects of perspective]. Paris: Dalmont et Dunod.

Meister, R. (1966). The iso-deformation of images and the criterion for delimitation of the usable areas in cine-auditoriums. Journal for the Society of Motion Picture \& Television Engineers, 75, 179-182

PERKINS, D. N. (1972). Visual discrimination between rectangular and nonrectangular parallelopipeds. Perception \& Psychophysics, 12. $396-400$.

Perkins, D. N. (1973). Compensating for distortion in viewing pictures obliquely. Perception \& Psychophysics, 14, 13-18

Perrone, J. A. (1980). Slant underestimation: A model based on the size of the viewing aperture. Perception, 9, 285-302.

Pirenne, M. H. (1970). Optics, painting, \& photography. Cambridge, England: Cambridge University Press.

Rhodes, G., Brennan, S. E., CARey, S. (1987). Identification and ratings of caricatures: Implications for mental representations of faces Cognitive Psychology, 19, 473-497.

Richter, J. P. (Ed. \& Trans.) (1970). The notebooks of Leonardo da Vinci. New York: Dover. (Original work published 1883)

RosINSKI, R. R., FARBER, J. (1980). Compensation for viewing point in the perception of pictured space. In M. A. Hagen (Ed.), The perception of pictures (Vol. 1, pp. 137-176). New York: Academic Press

Rosinskı, R. R., Mulholland, T., Degelman, D., \& Farber, J. (1980). Picture perception: An analysis of visual compensation. Perception \& Psychophysics, 28, 521-526.

SedGWICK, H. A. (1986). Space perception. In K. R. Boff, L. Kaufman, \& J. P. Thomas (Eds.), Handbook of perception and human performance (Vol. 1, pp. 21:1-57). New York: Wiley.

Thompson, P., DAvenPoRt, P. (1980). The dictionary of graphic images. New York: St. Martin's Press.

TODD, J. T., \& REICHEL, F. (in press). The visual perception of smoothly curved surfaces from double projected contour patterns. Journal of Experimental Psychology: Human Perception \& Performance.

WALLACH, H., MARSHALL, F. J. (1986). Shape constancy in pictorial representation. Perception \& Psychophysics, 39, 233-235.

Wallach, H., S Slaughter, V. (1988). Viewing direction and pictorial representation. Perception \& Psychophysics, 43, 79-82.

(Manuscript received September 27, 1989; revision accepted for publication January 9, 1990.) 